\title{
Differential Diagnosis Approach to Bilateral Peripheral Facial Paralysis: A Case Report
}

\author{
Bir Olgu Sunumu Eşliğinde Bilateral Periferik Fasiyal Paralizide Ayırıcı Tanı Yaklaşımı
}

\author{
Nihan Hande Akçakaya1, Meltem Hale Alpsan Gökmen², Yeşim Gülşen Parman³, Feza Deymeer³, Piraye Oflazer ${ }^{4}$ \\ 1 İstanbul University, Institute of Experimental Medicine, Division of Genetic, İstanbul, Turkey \\ ${ }^{2}$ Anadolu Health Center, Clinic of Neurology, İstanbul, Turkey \\ ${ }_{3}^{3}$ stanbul University İstanbul Faculty of Medicine, Department of Neurology, Science of Neuromuscular Diseases, İstanbul, Turkey \\ ${ }^{4}$ Memorial Şişli Hospital, Clinic of Neurology, İstanbul, Turkey
}

Keywords: Bilateral peripheral facial palsy, facial diplegia, Lyme disease, Guillain-Barre syndrome

Anahtar Kelimeler: Bilateral periferik fasiyal paralizi, fasiyal dipleji, Lyme hastalığı, Guillain-Barre sendromu

Dear Editor,

Bilateral facial paralysis (BFP) is described as involvement of the contralateral face within 30 days of the initial unilateral facial paralysis. This is a rare clinical presentation for which GuillainBarré syndrome syndrome (GBS) should first be considered. Exclusion of GBS often reveals an underlying systemic disease. BFP can be identified as idiopathic facial paralysis (Bell's palsy) only as a diagnosis of exclusion (1).

A man aged 60 years presented to our outpatient-clinic with facial immobility, inability to close his eyes or move his mouth, and hand paresthesia. His symptoms started 3 weeks previously with severe pain that radiated from the right shoulder to arm, and paresthesia localized to the fingertips within days. A week later, same symptoms repeated on the left. Facial paralysis occurred first on the left, then on the right side 3 days later. A physical examination revealed bilateral grade 4 (House-Brackmann) peripheral facial paralysis, bilateral hypoesthesia and hypoalgesia on hand fingertips, remarkable deep-sensation deficit in lower extremities, and loss of Achilles reflex on the left. The systemic examination was normal. Laboratory examinations including complete blood count, peripheral smear, blood biochemistry, erythrocyte sedimentation rate, and level of vitamin B12 were normal.

Electromyography (EMG) findings implied an early radiculopathy that caused conducting deficits in bilateral facial and peripheral nerves, and nerve roots. Also, a chronic involvement of the tibial division of the left sciatic nerve was identified. Lumber puncture revealed clear and colorless cerebrospinal fluid (CSF), and examination of the sample revealed $64 / \mathrm{mm}^{3}$ lymphocyte and $6 / \mathrm{mm}^{3}$ polymorphonuclear leukocytes. Serum anti-GQ1b and anti-GM1 were negative. Serum ACE levels were normal. Thorax computed tomography and brain magnetic resonance imaging (MRI) were normal. Lupus anticoagulants, RF, ANA, ENA screen, c-ANCA, p-ANCA, anti-dsDNA, anti-HIV, HTLV1, VDRL, and Brucella tube agglutination (Wright) tests yielded negative results. No uveitis was identified in ocular examination. Serum and urine immunofixation were normal. Direct X-rays of the humerus, cranium, and pelvis were also normal. Borrelia burgdorferi immunoglobulin (Ig) IgM was normal, but IgG was $187 \mathrm{RU} / \mathrm{mL}$ (>22 RU/mL positive). Accordingly, the patient was diagnosed as having Lyme disease, and motor and sensorial radiculoneuritis accompanied by cranial neuropathy due to Lyme

Address for Correspondence/Yazışma Adresi: Nihan Hande Akçakaya MD, İstanbul University, Institute of Experimental Medicine, Division of Genetics, İstanbul, Turkey

Phone: +9021241420 00-33318 E-mail: nhakcakaya@gmail.com

Received/Geliş Tarihi: 19.02.2016 Accepted/Kabul Tarihi: 19.06 .2016

${ }^{\circledR}$ Copyright 2016 by Turkish Neurological Society

Turkish Journal of Neurology published by Galenos Yayınevi. 
disease. Doxycycline therapy was administered for 21 days, at 100 mg twice-a-day dosage. With the exception of the loss of Achilles reflex, the clinical examination findings improved completely at three months.

Facial paralysis might have several etiologic causes including infectious, traumatic, neurologic, metabolic, neoplastic, toxic, vascular, and idiopathic causes. Further examinations should be performed in cases of bilateral involvement, concomitant involvement of other cranial nerves, prolonged facial involvement beyond one week or failure to improve within 3 weeks (2). More than half of unilateral peripheral facial paralysis cases have idiopathic etiology; idiopathic causes constitute less than $20 \%$ of bilateral cases. Table 1 summarizes the differential diagnosis of BFP in adults $(2,3)$.

The differential diagnosis of BFP should start with the medical history. The onset of paralysis and any prior symptoms, history of recent infection, trauma, otologic symptoms, changes in taste sensation, facial paresthesia, rashes, vaccinations, and weight loss should be questioned in the medical history.

Examinations should be performed considering medical history and potential life threatening diseases such as malignancy and GBS. A progressive weakness occurs in muscles innervated by peripheral and cranial nerves due to an autoimmune pathology in GBS. The facial nerve is involved in $27 \%$ to $50 \%$ of all cases (4). Approximately half of patients who present with facial nerve involvement are bilateral (3). GBS was suspected in our patient due to paresthesia at the fingertips, diminished deep sensation, and

\begin{tabular}{|ll|}
\hline $\begin{array}{l}\text { Table 1. Differential diagnosis of acquired bilateral facial } \\
\text { paralysis in adults* }(2,3)\end{array}$ & Guillain-Barre syndrome \\
Neurologic & Multiple sclerosis \\
& Pseudobulbar and bulbar paralysis \\
Trauma & Cranium fractures \\
& Parotid surgery \\
& Mastoid surgery \\
Infections & Post-influenza \\
& Infectious mononucleosis \\
& HIV infection \\
& Lyme disease \\
& Syphilis \\
& HTLV1 infection \\
& Brain stem encephalitis \\
& Poliomyelitis \\
*The table has been adapted from & Diabetes \\
Metabolic & Acute porphyria \\
Adiopathic & Acoustic neurinoma \\
Neoplastic & Central nervous system metastases \\
& Acute leukemia \\
& Multiple myeloma \\
& Sarcoidosis \\
& Amyloidosis \\
& \\
&
\end{tabular}

loss of reflexes. However, classic GBS was excluded because of the CSF examination results, although EMG findings were supportive. Malignancy and infectious etiologies were primarily investigated in our case due to the identification of cells in CSF examination and advanced age. Examination of patients who present with BFP should encompass cranial MRI and investigations aimed at diagnosing hematologic malignancy and multiple myeloma that might infiltrate peripheral and cranial nerves. Erythrocyte sedimentation rate, HIV, Lyme, Brucella, syphilis, and HTLV1 tests should be planned in terms of infectious diseases. Other diseases to be considered in the differential diagnosis include diabetes, sarcoidosis, and rheumatologic diseases. Fasting glucose, serum ACE level, and antinuclear antibody level should also be examined $(2,3)$. Our patient was diagnosed as having Lyme disease because of the elevated Lyme titers, despite the absence of tick bite, rash or erythema migrans (EM) in the medical history.

Lyme disease, caused by a spirochete called Borrelia burgdorferi, is the most common infectious cause of BFP (5). EM might be absent in $10 \%$ of patients, and patients might be asymptomatic or only have nonspecific symptoms in the early disease period (5). Neurologic involvement is seen in 15-20\% of Lyme cases and facial paralysis is the most common form of neurologic presentation $(5,6)$. CSF pleocytosis with predominant lymphocytes accompanied by normal or elevated CSF protein level is seen in central nervous system involvement (6). Similar to our case, a painful radiculopathy might accompany facial paralysis (6). Identification of Borrelia burgdorferi antibody both in CSF and serum is the most specific diagnostic test in suspected cases with central nervous system involvement of Lyme disease (6). Treatment should be initiated following establishment of a serologic diagnosis. Cardiovascular involvement, encephalopathy, polyneuropathy, radiculomyelitis, and chronic oligoarticular arthritis might develop in cases that are left untreated. The objective of treatment is to prevent advanced complications associated with Lyme disease $(5,6)$.

Unlike Bell's palsy, BFP often results from an underlying systemic or general neurologic pathology, and prog-nosis depends on the underlying etiology. Further examinations should be performed before prescribing Bell's palsy treatment, and treatable causes including Lyme disease as well as other life-threatening diseases should be considered in the differential diagnosis.

\section{Ethics}

Peer-review: Externally and internally peer-reviewed.

\section{Authorship Contributions}

Surgical and Medical Practices: Nihan Hande Akçakaya, Meltem Hale Alpsan Gökmen, Piraye Oflazer, Yeşim Gülşen Parman, Feza Deymeer, Concept: Nihan Hande Akçakaya, Meltem Hale Alpsan Gökmen, Design: Nihan Hande Akçakaya, Piraye Oflazer, Data Collection or Processing: Meltem Hale Alpsan, Yeşim Gülşen Parman, Analysis or Interpretation: Nihan Hande Akçakaya, Meltem Hale Alpsan Gökmen, Piraye Oflazer, Yeşim Gülşen Parman, Feza Deymeer, Literature Search: Nihan Hande Akçakaya, Meltem Hale Alpsan Gökmen, Piraye Oflazer, Feza Deymeer, Writing: Nihan Hande Akçakaya, Piraye Oflazer.

Conflict of Interest: No conflict of interest was declared by the authors.

Financial Disclosure: The authors declared that this study received no financial support. 


\section{References}

1. Adour KK, Byl FM, Hilsinger RL, Jr., Kahn ZM, Sheldon MI. The true nature of Bell's palsy: analysis of 1,000 consecutive patients. Laryngoscope 1978;88:787-801.

2. Morrow MJ. Disorders of the Facial Nerve. Neurological Therapeutics Principles and Practice. In: Noseworthy JH, Rochester MN (eds). 2nd ed. USA, 2006:2108-2115.
3. Jain V, Deshmukh A, Gollomp S. Bilateral facial paralysis: case presentation and discussion of differential diagnosis. J Gen Intern Med 2006;21:7-10.

4. May M. The Facial Nerve. New York: Thieme Inc, 1986:181.

5. Lay M. The Facial Nerve. In: Jster MN (ed). 181sentation and discussion of differential diagnosis. 2nd ed. USA, 2006:1025-1038.

6. Aksamit AJ. Peripheral Nervous System Lyme Disease. In: Noseworthy JH, Rochester MN (eds). Neurological therapeutics principles and practice. 2nd ed. USA, 2006:2229-2231. 